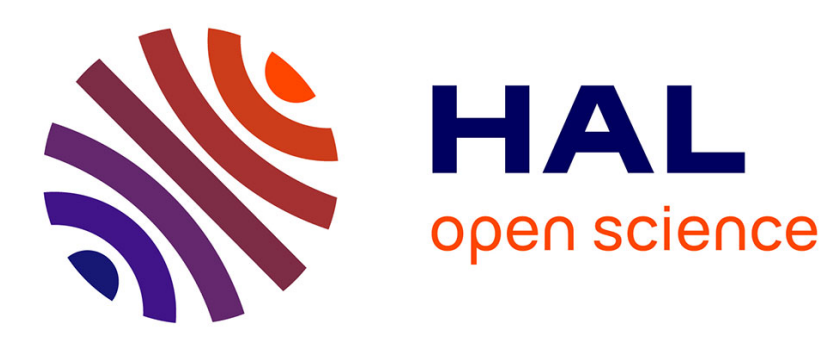

\title{
Sustained inertial-capillary oscillations and jet formation in displacement flow in a tube
}

\author{
Yi Sui, Peter D.M. Spelt
}

\section{To cite this version:}

Yi Sui, Peter D.M. Spelt. Sustained inertial-capillary oscillations and jet formation in displacement flow in a tube. Physics of Fluids, 2011, 23, pp.122104. 10.1063/1.3670010 . hal-00691093

\section{HAL Id: hal-00691093 \\ https://hal.science/hal-00691093}

Submitted on 11 Jun 2012

HAL is a multi-disciplinary open access archive for the deposit and dissemination of scientific research documents, whether they are published or not. The documents may come from teaching and research institutions in France or abroad, or from public or private research centers.
L'archive ouverte pluridisciplinaire HAL, est destinée au dépôt et à la diffusion de documents scientifiques de niveau recherche, publiés ou non, émanant des établissements d'enseignement et de recherche français ou étrangers, des laboratoires publics ou privés. 


\title{
Sustained inertial-capillary oscillations and jet formation in displacement flow in a tube
}

\author{
Yi Sui ${ }^{1}$ and Peter D. M. Spelt ${ }^{1,2}$ \\ ${ }^{1}$ Department of Chemical Engineering, Imperial College London, London, United Kingdom \\ ${ }^{2}$ Laboratoire de Mécanique des Fluides et d'Acoustique, CNRS, Ecole Centrale de Lyon, Ecully, \\ and Département Mécanique, Université Claude Bernard Lyon 1, France
}

(Received 1 April 2011; accepted 8 November 2011; published online 16 December 2011)

\begin{abstract}
We study inertial effects in the displacement of a fluid in a capillary by a more viscous fluid, using a numerical method. A level-set approach is employed to track the meniscus, and a Navier slip boundary condition is imposed in order to alleviate a stress singularity at the moving contact line. Various flow regimes are identified with a Reynolds number and a capillary number as the main parameters. At relatively low Reynolds number, the meniscus forms a steady shape, and the interfacial curvature at the tube centre can change from being concave to convex upon increasing the Reynolds number if the displacing fluid is wetting to the tube surface. For wetting displacing fluids, beyond a critical Reynolds number, a quasi-steady solution is no longer found: instead, the interface undergoes non-dampened periodic oscillations and, at even larger values of the Reynolds number, quasi-periodically, and the interface evolves from simple wavy shapes to complex shapes with multiple wavy units. This oscillating state is observed for sufficiently small contact angle values defined from the displacing fluid $\left(<80^{\circ}\right)$. Beyond a second critical Reynolds number, the displacing fluid forms a jet and the meniscus advances with a nearly constant speed which decreases with $R e$. This is also observed at large contact angle values. In a developing jet, however, the interface shape remains partially quasi-steady, near the contact line region and the tube centre. The flow behaviour is shown to be robust over a range of other governing parameters, including the capillary number and the slip length. The potential implications of the work on network models of two-phase flow through porous media are discussed. (C) 2011 American Institute of Physics. [doi:10.1063/1.3670010]
\end{abstract}

\section{INTRODUCTION}

Flow wherein one fluid displaces another in a tube serves as a model for many phenomena in nature and industry, such as in oil and gas transport through porous media. In a single capillary, studies of steady and transient displacement flows have been conducted for cases where the displaced fluid is much more viscous or even non-Newtonian (e.g., Refs. 1-5). Network models of immiscible displacement in porous media consist of such capillaries as building blocks on the pore scale, often revealing fingering phenomena (e.g., Refs. 6-13). Often Stokes flow is assumed by virtue of the small pore sizes. In capillaries, the meniscus shape is then taken to be a circular cap consistent with an apparent contact angle, and the corresponding pressure drop is used. Single-pore studies have shown that this is a reasonable assumption as long as the capillary number is sufficiently low (see, for instance, Ref. 14, as briefly reviewed further below). Inertial effects in the rise of a liquid in a capillary towards an equilibrium liquid column height have also been studied extensively, and it is well established that these take the form of inertial-capillary oscillations in the height of the liquid column about its equilibrium state that dampen out in time (e.g., Refs. 15 and 16; a comprehensive model of this behaviour is presented in Ref. 17). Here, we are concerned with inertial effects in capillary flows through tubes under conditions, whereas the flow rate of displacing fluid is fixed instead, and not the result of capillary action, hence inertial oscillations in the total volume occupied by the displacing fluid cannot occur. As yet, it is unclear what inertial effects would occur here then. This "capillary flow that is not entirely due to capillarity" is also of interest when considering high-speed two-phase flow through porous media (reviewed in Ref. 18, including cases whereas the Reynolds number based on pore size was larger than $10^{3}$ ). There, the single-phase equivalent flow has received much attention so far, centred mainly around extensions of a linear Darcy law between pressure gradient and flow rate, by coupling the flow on the micro (or pore) scale to the macroscopic description (e.g., Refs. 19-25).

In the present study, we demonstrate that inertia first enters through modification of the steady shape of the meniscus at modest values of the Reynolds number, Re. Beyond this, sustained oscillations in the shape of the front of the displacing fluid may occur through a supercritical Hopf bifurcation, which is characterized herein as well. The oscillations are not in the height of the liquid column (studied extensively in, e.g., Refs. 15-17), but in the velocity of the contact line and the centreline velocity of the meniscus. A subsequent jet-type flow regime, observed beyond a second critical value of $R e$, is also investigated and characterized. Finally, the robustness of these regimes in terms of other dimensionless groups is established. The requirement of inertial effects for the existence of these flow regimes is discussed in Sec. IV.

We briefly discuss the prior work on the quasi-steady flow regime here first. A majority of experimental studies ${ }^{26-28}$ 
have focussed on measuring an apparent dynamic contact angle and establishing its relation with the static contact angle and speed of the contact line, to test classical theories ${ }^{29-31}$ of moving contact lines. Finite-element simulations, with a Navier-slip boundary condition (to alleviate the stress singularity at the contact line), have also been carried out to study the steady macro meniscus shapes, for different values of the slip length and capillary number in the creeping-flow regime. ${ }^{32,33}$ A theoretical model has been developed by Dussan et $a l .{ }^{34}$ to predict the interface shape near the moving contact line, where the inner region and outer region overlap. This approach was extended beyond its original region of validity to predict the macroscopic interface shape by Boender et al. ${ }^{35}$ and by Ramé ${ }^{14}$ for a liquid advancing or receding in a capillary tube filled with air.

Obviously, these earlier studies are all confined to low values of a Reynolds number, and an objective of the present investigation is to study the inertial effects in these systems as outlined above, allowing for unsteady flow regimes; the flow is assumed to be axisymmetric. A slip-length based level-set method, along the lines of that developed and well tested initially by Spelt, ${ }^{36,37}$ is adopted for this purpose. The problem statement and numerical method are summarized in Sec. II. The validation against prior work in the quasi-steady flow regime, and further analysis of this regime is discussed in Sec. III A. Results and analysis of a new oscillatory regime and a jetting regime are presented in Secs. III B and III C, respectively. The flow regimes are shown to occur over a range of capillary numbers and other parameters in Sec. III D. The findings are also summarized in the form of a map of flow states. Finally, we establish the significance of the present findings for an application in network models ${ }^{7,12,13}$ for flow through porous media by investigating the behaviour of a relative flow resistance.

\section{PROBLEM STATEMENT AND NUMERICAL METHOD}

As illustrated in Fig. 1, we shall consider one fluid, which is pumped into a tube with cross-sectional radius, $a$, displaces another fluid with which the tube is filled initially. The contact angle $\vartheta$ is defined from the displacing fluid and its value is kept constant. Such an assumption has been used in theories ${ }^{14,29-31}$ and numerical simulations ${ }^{32,33}$ of displacement flows, drop spreading, and sliding. For most cases in the present study, $\vartheta=45^{\circ}$ is used, which corresponds to wetting displacing fluids, although the effect of changing the contact angle is studied in detail in Sec. III D. Both fluids are incompressible, viscous, and immiscible. The viscosity and density ratios of the fluids are denoted as $\lambda_{\mu}=\mu_{2} / \mu_{1}$ and $\lambda_{\rho}=\rho_{2} / \rho_{1}$, respectively. In the present discussion, we consider mainly the case $\lambda_{\mu}=\lambda_{\rho}=0.05$, tests have shown that further decreasing these ratios to 0.01 results in quantitatively very similar results. We assume that the problem is axisymmetric, and the size of the computational domain is $a \times 72 a$, which has been found long enough to capture the various flow states.

At the inlet $(z=0)$, fully developed Poiseuille flow is imposed with a cross-sectionally averaged speed $U$. At the outlet, a zero normal velocity gradient outflow condition is

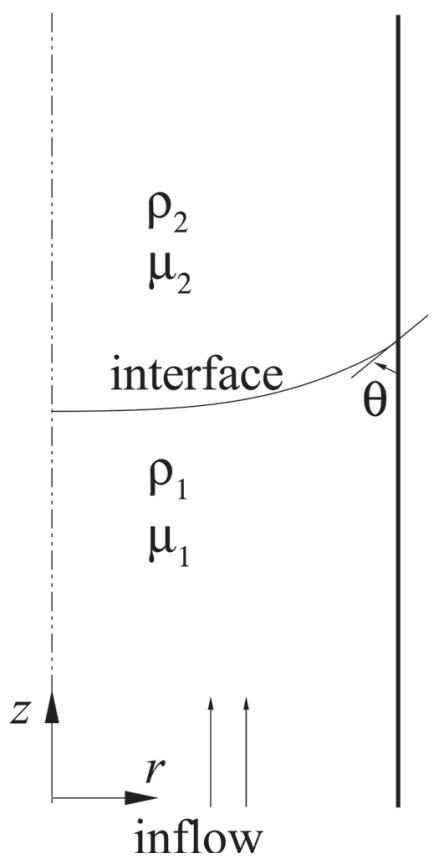

FIG. 1. Problem definition sketch.

used. At the centreline of the tube, a symmetry boundary condition is used and at the wall $(r=a)$, a Navier slip boundary condition, $U_{w}=-\varepsilon \partial u_{z} / \partial r$, is employed, where $\epsilon$ represents the slip length. In the present study, the tube radius $a$ and the average flow velocity $U$ are chosen as the macroscopic length and velocity scales, respectively. Hence, the Reynolds number is defined as $R e=\rho_{1} U a / \mu_{1}$, and the capillary number as $C a=\mu_{1} U / \sigma$, where $\sigma$ represents the interfacial tension between the two fluids. The initial velocity condition in the tube is set as fully developed Poiseuille flow. At low Reynolds number values, the initial interface profile is flat; this evolves into a deformed, but steady-state shape (discussed further below) that we have found to be independent of the initial interface shape used, for example, a steady wavy shape from a simulation with a lower Reynolds number. At high Reynolds numbers, the interface cannot achieve a steady state, and the initial shape is set as the shape of the highest Reynolds number for which a steady state could be achieved (specified further in Sec. III).

The level-set method used herein is essentially that of Spelt, ${ }^{36,37}$ and Ding et al., ${ }^{38,39}$ which is an extension of the method developed by Sussman et al. ${ }^{40}$ to account for moving contact lines. Here, we just give a brief summary (details can be found in the pertinent literature).

In level-set methods, the level-set function $\varphi(\mathbf{x}, t)$ is taken to be a signed distance function from the interface, and the sign is used to distinguish between the two fluids; the zero level set corresponds to the interface. The level-set function is advanced using the advection equation,

$$
\frac{\partial \varphi}{\partial t}+\boldsymbol{u} \cdot \nabla \varphi=0
$$

The advection equation only holds at $\varphi=0$, and a "redistance" step ${ }^{41}$ is used to ensure that $\varphi$ at points away from the interface remains approximately the signed distance 
function. The equations of motion are combined into one variable-density/viscosity formulation, with the local density and viscosity are functions of $\varphi(\mathbf{x}, \mathrm{t})$. The abrupt change in these fluid properties across the interface is smoothed out over a length that is proportional to the grid spacing. The action of surface tension is represented as a singular force density term in the momentum equation, also smoothed out over several mesh points.

In the present simulation, a finite volume method based on a marker-and-cell (MAC) mesh is employed. The velocity components are defined at cell faces and scalar variables such as pressure and volume fraction are defined at the cell centres. A fifth-order weighted essentially non-oscillatory (WENO) scheme $^{42}$ is employed in the discretization of the advection term in level-set function using the local flow velocity as the up-winding direction. More details regarding the implementation of boundary condition to account for moving contact lines with a specified contact angle can be found in Spelt ${ }^{36,37}$ and Ding et al., ${ }^{38,39}$ where various numerical tests have been carried out. For all cases considered in the present study, we have found that the global error in mass conservation for each phase is below $0.1 \%$ throughout the simulations.

Most interface capturing methods employ a MAC grid, such that the volume fraction is advected by the cell-facenormal velocity. This induces a numerical slip when simulating moving contact line problem. ${ }^{43,44}$ However, it is found that when the slip length is resolved by the mesh resolution, the results converge. This greatly restricts the slip length one can simulate, though in most of our simulations we use a slip length of $0.02 a$ for this reason. This is further discussed in Sec. III D. Finite-element methods, ${ }^{32,45,46}$ with local mesh refinement have been employed previously and much smaller slip lengths have been simulated, but those were for steady flows or flows without contact lines. In the present study, a grid size of $51 \times 3601$ has been employed to cover the computational domain, and we find that this is sufficient to give a converged result. Tests on the temporal evolution of the contact line location along the tube wall and the instantaneous interface shapes gave nearly identical results for $d r=0.01 a$ and $0.02 a$ (not shown).

\section{RESULTS AND DISCUSSION}

In the present investigation, unless indicated otherwise, we change the Reynolds number whilst keeping all other parameters constant. For the cases considered, in general, we have indentified three flow states: (a) a quasi-steady state where the meniscus reaches a steady shape and the contactline speed is constant; (b) an oscillating state wherein the interface shows non-dampened oscillations; (c) a jetting state. Details of the various states are presented and discussed separately in Secs. III A-III C below, followed by a further parametric study.

\section{A. Steady regime}

For $C a=0.05, \vartheta=45^{\circ}$, we find that the meniscus reaches a steady shape when the flow Reynolds number is relatively small after an initial transient stage. The steady meniscus shapes for different Reynolds numbers are presented in Fig. 2(a), where we have made the respective locations of the contact line to coincide for convenience of presentation. The steady, concave menisci for $R e=0.5$ and 5 are virtually identical. A matched asymptotic approach for creeping flows has been used previously, ${ }^{14,34,35}$ to predict the meniscus advancing or receding in a capillary tube. These theoretical results have been found previously to agree very well with finite elements simulation by Lowndes. ${ }^{32}$ For comparison, we have also plotted the steady interface shape predicted by this theoretical model ${ }^{14}$ in Fig. 2(a); it is seen that the present simulations also agree well with this previous result. With increasing Reynolds number, the interface shape predicted by the simulations obviously deviates from the analytical solution, which does not account for any inertia effects. An interesting feature here is that the interface at the tube centre changes from concave to convex (the curvature changes sign); the transition happens between $R e=50$ and 100. This is clearer in Fig. 2(b), which shows the angle between interface and vertical tube wall as a function of interfacial distance to the moving contact line. The distance to the moving contact line has been normalized by the total length of the curved interface. For creeping flow, along the interface and apart from the contact line, the angle increases monotonically while at larger Reynolds

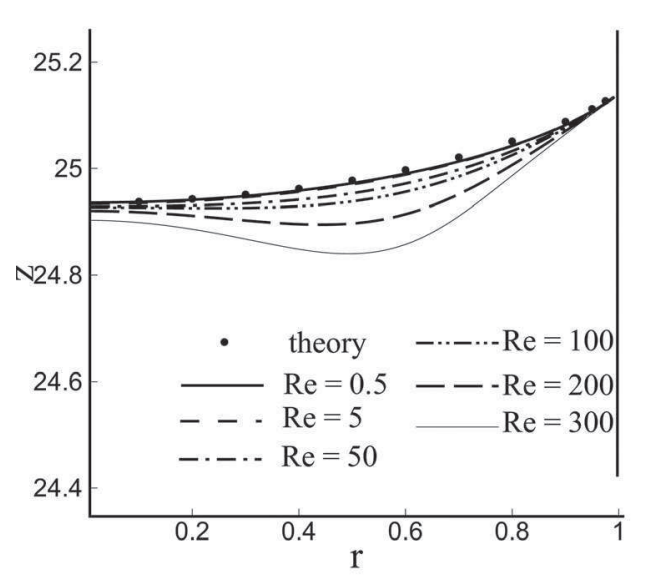

(a)

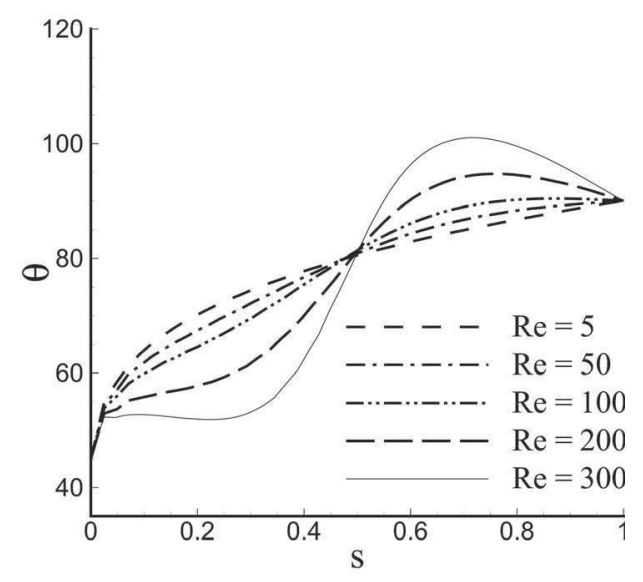

(b)
FIG. 2. (a) Steady interface shapes and (b) angle between interface and vertical tube wall as a function of interfacial distance to the moving contact line. 


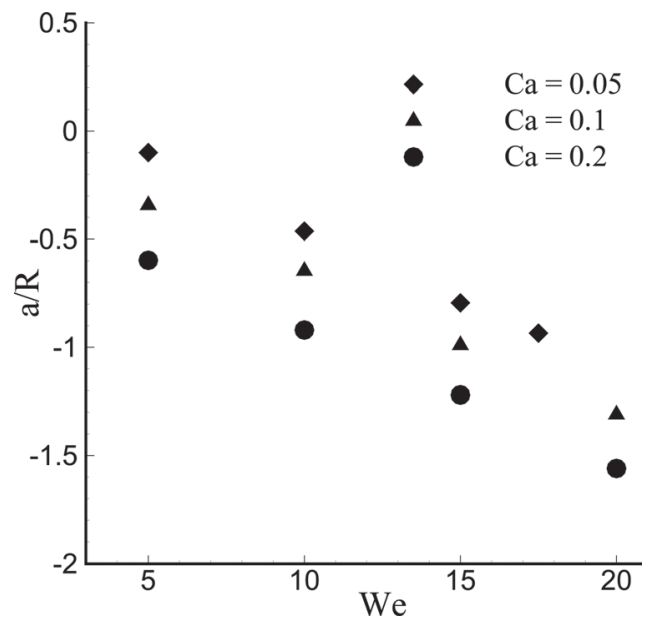

FIG. 3. Curvature of spherical cap at the tube centre as function of Weber number.

numbers, the angle increases to a value large than $90^{\circ}$ and then decreases to $90^{\circ}$, indicating that the meniscus has a wavy shape.

A simple force balance can be formulated at these values of $R e$ (especially when far removed from the contact line), by equating the orders of magnitude of inertia and capillary forces, $\rho U^{2} \cdot R^{2}$ and $(\sigma / R) \cdot R^{2}$, where $R$ is the radius of the spherical cap at the tube centre. This gives $a / R \sim W e$ (where $W e=\operatorname{Re}(a)$. In Fig. 3, we present the normalized curvature of the spherical cap at the tube centre as a function of Reynolds number for cases with different capillary numbers, and it can be seen that $a / R$ changes nearly linearly with $W e$.

The flow pattern corresponding to the quasi-steady state is shown in Fig. 4. It can be seen that the interface forms a streamline, as expected. In the contact line region, the typical wedge flow pattern is observed, as predicted by Cox. ${ }^{29}$ The splitting ejection streamline is seen to lie in the less viscous (upper) fluid, and this is found to enclose a ring vortex above the meniscus.

\section{B. Oscillating regime}

Here, initially we have started simulations with the steady-state meniscus shape and flow field obtained for

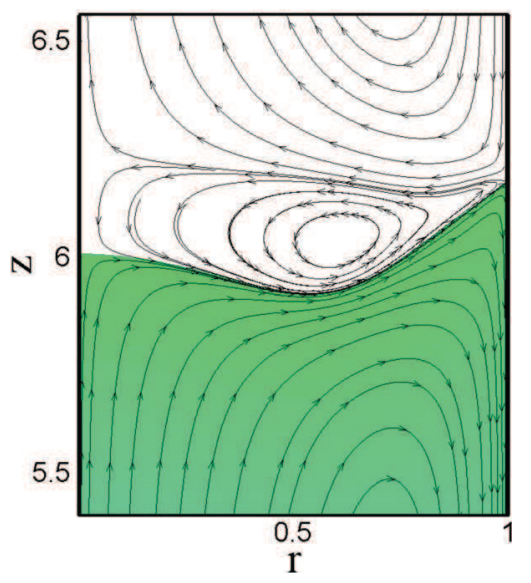

FIG. 4. (Color online) Flow field around the interface for $R e=300$ (in a frame of reference moving with the contact line).
$R e=350$. When the Reynolds number is now increased above a critical value, around 365 for the present case, a quasi-steady solution is no longer found. Instead, a periodic oscillation is observed. We have observed hysteresis in the transition, that is, if a simulation is started from an instantaneous interface shape and flow field around at $R e=400$, we find that the flow becomes steady when the Reynolds number is less than 360. For the results presented in this section, we use the latter as the initial condition.

The properties of the periodic state are presented in Fig. 5 for $R e=400$. As can be seen in Fig. 5(a), the contact line velocity along the tube wall evolves with time and its Fourier power spectrum shows one fundamental frequency and its harmonics. The meniscus shape also oscillates at this frequency. The meniscus shapes within one period are shown in Fig. 5(c).

The oscillation amplitude of the contact line velocity along the tube wall is presented as a function of Reynolds number in Fig. 6. It can be seen that just after the transition from the steady to the periodic state, the amplitude increases with Reynolds number nearly linearly. Since any possible quasi-steady solution at these Re values would be unstable, this suggests a supercritical Hopf bifurcation.

Further increase of the Reynolds number leads to a quasiperiodic state, as shown, for example, in Fig. 7 for $R e=500$. A Fourier transformation has been carried out for the temporal evolution of the contact line velocity along the tube wall and the power spectra, the result of which is shown in Fig. 7(b). Two fundamental frequencies are observed as well as the linear combination $m f_{1}+n f_{2}$, where $m$ and $n$ are integers, $f_{1}$ and $f_{2}$ are the high and low fundamental frequencies, respectively. The instantaneous interface profiles are shown in Figure 7(c), at $t=0.326 T_{1}$ and $0.489 T_{1}$, it can be seen that along the interface the curvature changes sign twice. In the periodic state with one fundamental frequency, the interfacial curvature only changes sign once along the meniscus.

If the interface is modelled as a spring with the spring stiffness being the coefficient of surface tension, the natural response time would be the inertial/capillary time scale $T=\sqrt{\rho a^{3} / \sigma}$ (as pointed out previously by Biance et $a l^{47}$ for liquid drops, for example). Figure 8 shows the oscillation period of the contact line velocity along the tube wall, normalized by the inertia/capillary time scale, as a function of Reynolds number. The results suggest that the dynamical behaviour of the interface can indeed be scaled by the inertia/capillary time. The capillary wave propagates between the contact line and tube centre. Bian et $a l .{ }^{48}$ studied the natural frequency of a liquid slug in capillary tube theoretically and experimentally. They found that the natural frequency is related to the axial length of the liquid mass. For the present tube flow, the liquid part is increasing its length continuously whereas the frequency and oscillation amplitude remain constant, which suggests that the present oscillation is different from that of Bian et al. ${ }^{48}$

\section{Jetting regime}

Beyond $R e=600$, the oscillation features of the interface disappear and the interface forms a jet instead. A good 


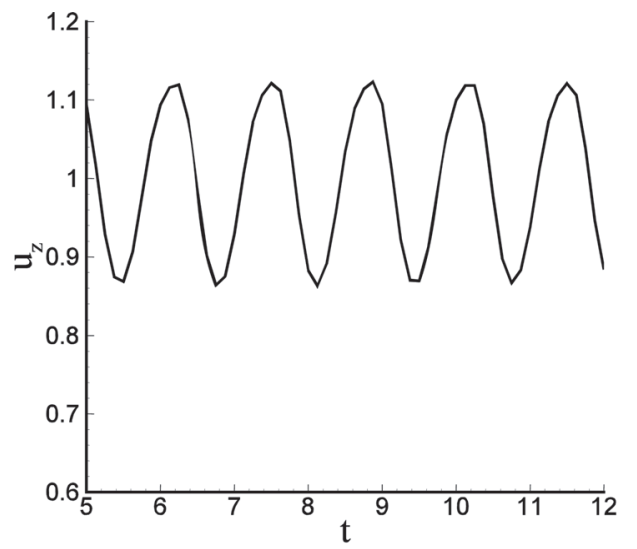

(a)

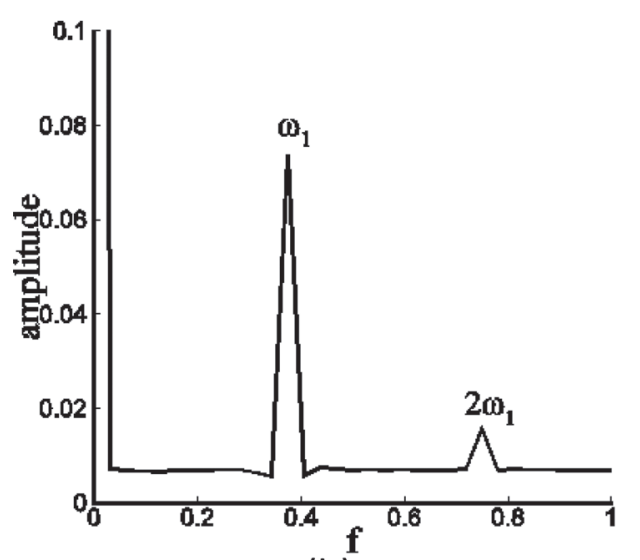

(b)

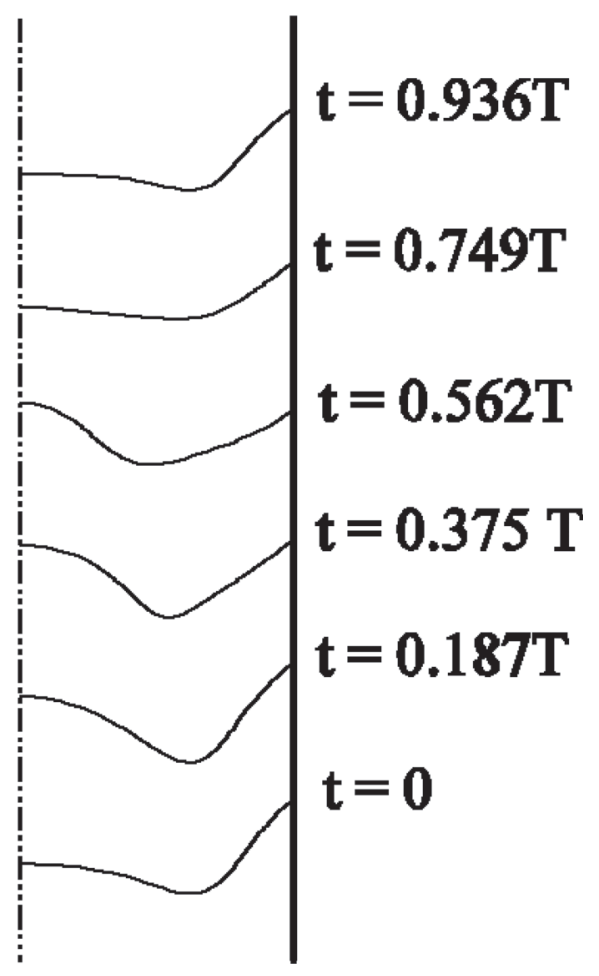

(c)
FIG. 5. (a) Time evolution of the contact line velocity along the tube wall; (b) Fourier power spectra of the contact line velocity; (c) interface shapes within an oscillation period for $R e=400$. example of this jetting regime is for $R e=800$, a snapshot of which is shown in Fig. 9. As can be seen from Fig. 9(a), most liquid flows along the tube centre and a slender liquid jet develops. Along the tube wall, a film of the less viscous fluid is formed and its length is increasing with time.

We investigate the instantaneous interface shape at different times by collapsing the contact line positions, and the results are presented in Fig. 9(b). It is interesting to see that there is a quasi-steady region of the interface around the contact line, in spite of the overall unsteady nature of this flow

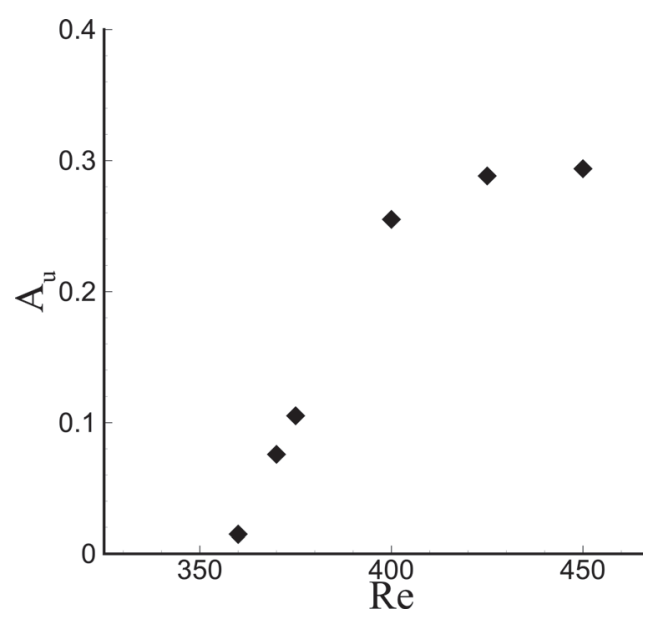

FIG. 6. Oscillation amplitude of the contact line velocity along the tube wall as a function of Reynolds number for $C a=0.05$. state. Similarly, the region around the tube centre is found to be quasi-steady. Between these two regions, the jet is increasing its length continuously.

Next, we investigate whether the jet length approaches a constant value, which would require that the velocity of the interface at the tube centre and the contact line must finally become the same. Figure 10(a) presents the temporal evolution of the interface velocity at these two locations. It can be seen that the contact line moves in a quasi-steady manner, in which the velocity is increasing extremely slowly. At the tube centre, the interface velocity decreases slowly and starts a slight oscillation at late times. This oscillation is due to a capillary-type instability which develops at the jet front. Fig. 10(b) presents the instantaneous interface profile at $t=40$, which is well before the velocities of the interface at the tube centre and contact line approach each other. It can be seen that a capillary-type instability becomes apparent in the necking region, and the bulge in the jet approaches the wall to form new contact lines (which is beyond the scope of the present work). We have observed similar phenomena for all cases that lead to a jetting regime.

We investigate the flow field around the moving contact line in the jetting regime, and present the result, in Fig. 11. Very close to the contact line, a wedge flow pattern similar to that in the steady regime is observed once more. We note however that the interfacial velocity (in the moving frame of reference) changes sign along the interface at a stagnation point. The stagnation point is in fact the boundary separating 


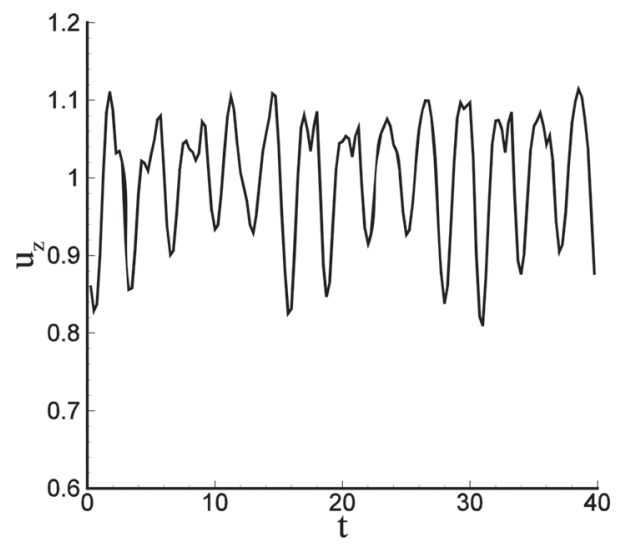

(a)

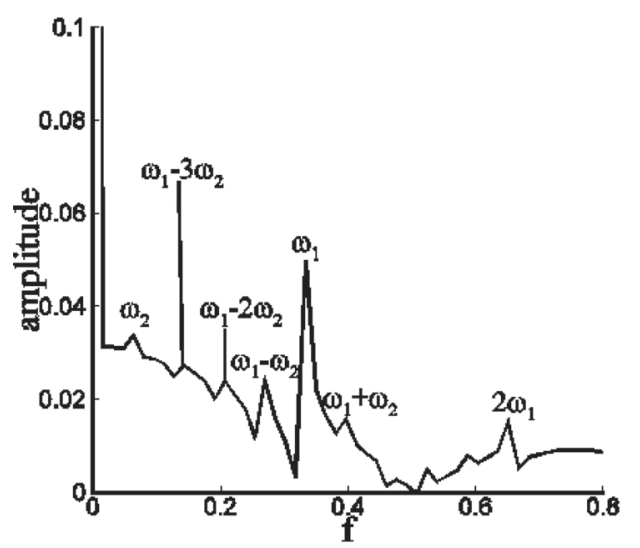

(b)

the quasi-steady region near the contact line from the middle region whose length is increased continuously by viscous shear force.

In the jetting regime, one can again fit a circular cap to the quasi-steady interface at the tube centre and present its curvature as function of Reynolds number; the result is shown in Fig. 12, which also includes the results from the steady regime. We find that $a / R$ changes linearly with $R e$ along the same line in both regimes. This further supports our force balance arguments at the tube centre presented in Sec. III A. In the other quasi-steady region of the interface around the moving contact line, we have also fitted a circle to the lowest part of the interface and determined its curvature. Alternatively this can be achieved by computing $d s / d \vartheta$ at $\vartheta=90^{\circ}$ near the contact line. The curvature is plotted in Fig. 13 as a function of Reynolds number. It can be seen that the curvature increases with $R e$. Actually, the radius of curvature of the quasi-steady region of the interface around the moving contact line is positively related to the thickness of the film between the jet and the wall. Our results show that, with increasing Reynolds number or capillary number, the film thickness decreases.

\section{Parametric study: Effects of $\theta, \lambda_{\mu}, \mathrm{Ca}$, and $\varepsilon$}

We have studied the effect of the value of the contact angle on the flow states in the tube. In the steady regime, it is found that when the contact angle is larger than $90^{\circ}$, the interface can still form a steady shape but is no longer wavy, that is, the curvature does not change sign along the interface. For higher values of the Reynolds number where the oscillation of the interface could appear, results for different contact angles are presented in Figure 14 for $R e=450$, $C a=0.05$. With increasing the contact angle, the oscillation amplitude of the contact line velocity decreases and a transition to jetting happens when the contact angle is beyond $80^{\circ}$. In conclusion, use of non-wetting displacing fluids promotes

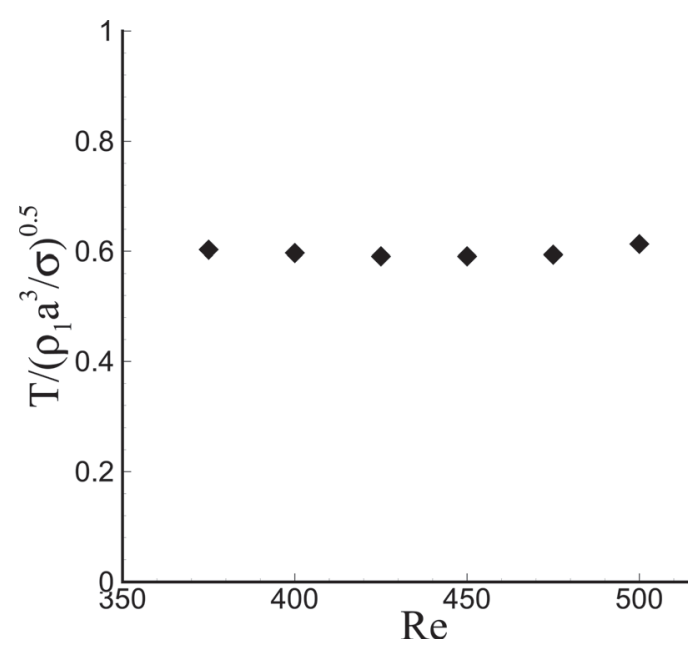

FIG. 8. Dimensionless oscillation period of the contact line velocity along the tube wall as a function of Reynolds number. For quasi-periodic state at $R e=500, \mathrm{~T}_{1}$ is used. 


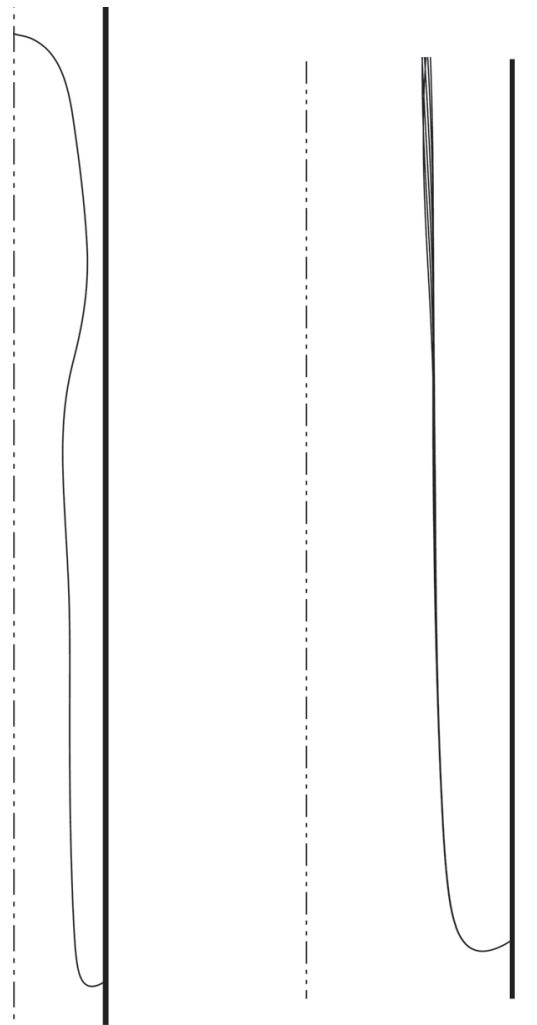

FIG. 9. For $R e=800$ (a) instantaneous interface profile at $t=25$; (b) interface profiles for $t=20,21,22,23,24$, and 25 around the contact line (we have collapsed the contact lines).

the formation of jets in displacement flows and suppresses inertial-capillary oscillations.

The effect of the value of the viscosity ratio on the transition to jetting has also been investigated and the results are presented for $\vartheta=45^{\circ}$ and $C a=0.05$ in Fig. 15. It can be seen that if the displacing fluid is less viscous, a jet tends to develop at lower Reynolds numbers. For example, at $\lambda_{\mu}=5$, the flow is already in a jetting regime when $R e$ is beyond 50 and oscillations of the interface are not observed.

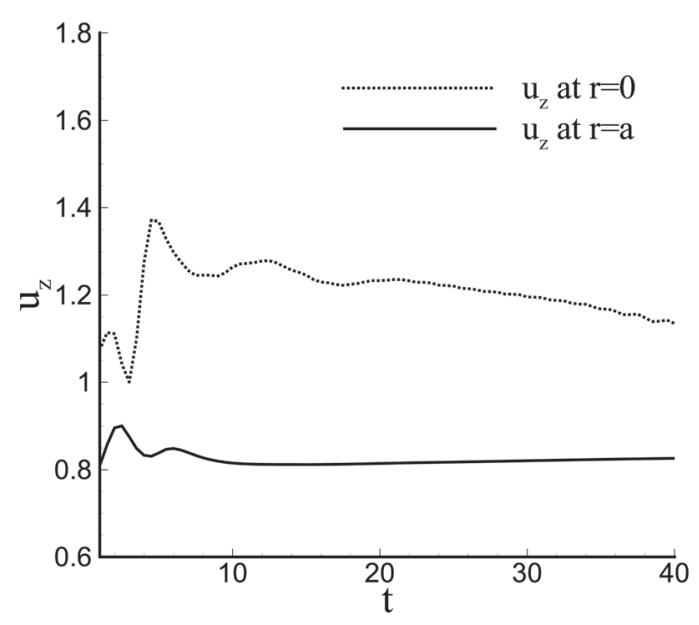

(a)

FIG. 10. (a) Temporal evolution of the interface velocity at the tube centre and the contact line; (b) interface profile at $t=40$ for $R e=800$.

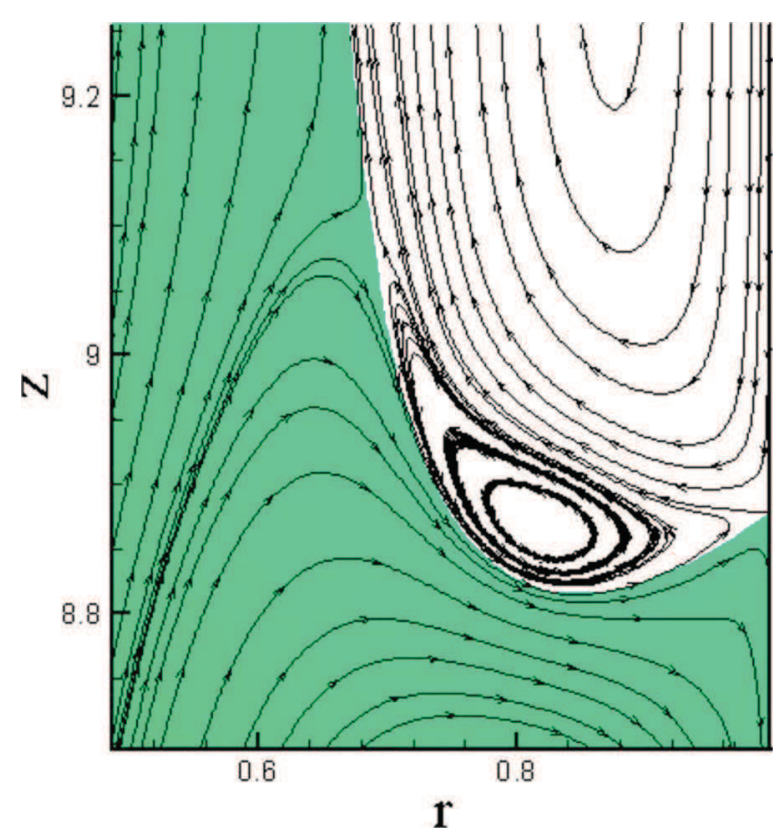

FIG. 11. (Color online) Streamline pattern around the contact line for $R e=800$ (in a frame of reference moving with the contact line).

We have also conducted simulations at different capillary numbers, from $C a=0.025$ to 0.2 , and a phase diagram of different flow states is shown in Fig. 16 for $\vartheta=45^{\circ}$, in terms of a Weber number $W e=R e C a$ versus $R e$, for convenience of presentation. It can be seen that the jetting regime is reached beyond a critical value of $W e$ close to 30 . The oscillatory state is observed beyond a critical value of $R e$, and to occur over an increasing range of $W e$ as $R e$ is increased.

A restriction of the present study is that the slip length $\epsilon=O\left(10^{-2}\right)$, which falls well short of realistic conditions which would demand $\epsilon=O\left(10^{-4}\right)$, at most. ${ }^{49,50}$ This is mainly due to the fact that in direct numerical simulations the slip length must be resolved by the numerical grid. A realistic slip length would require a mesh resolution that would be well beyond present computational resources. Even so,

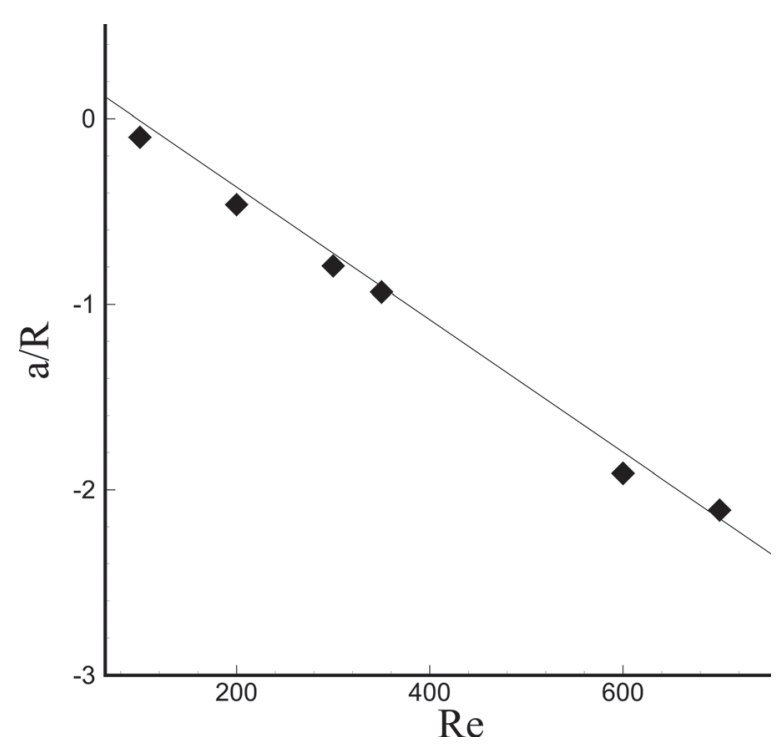

FIG. 12. Curvature of the spherical cap at the tube centre as function of Reynolds number. 


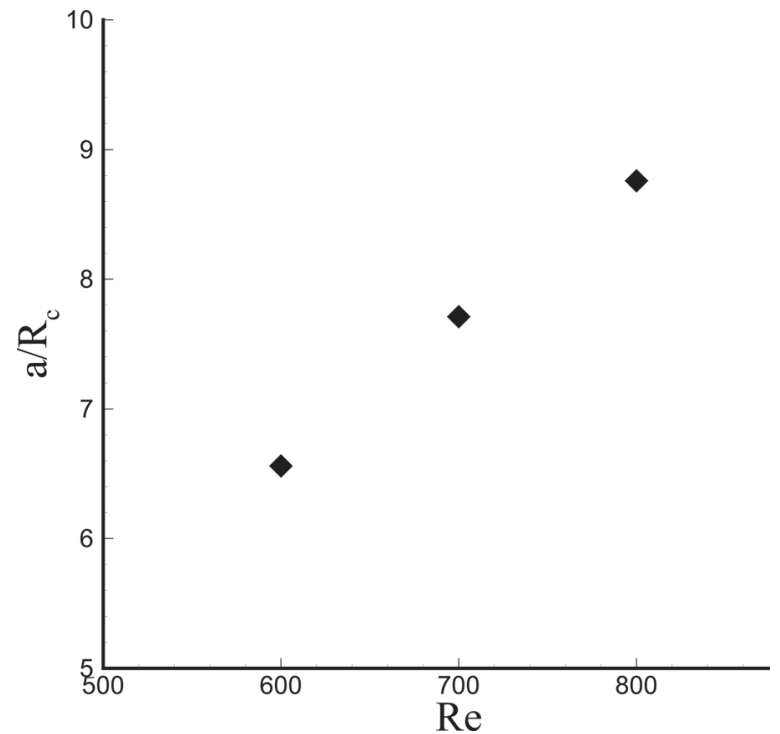

FIG. 13. Curvature of spherical cap near the contact line as function of Reynolds number.

we have carried out several simulations at a significantly smaller slip length value of $\epsilon=0.004$. The grid spacing was also reduced to $d r=0.004$, where results were found to have converged with respect to the grid spacing.

The time signals of the contact line velocity along the tube wall for different slip lengths are presented in Fig. 17 (a). It can be seen that lowering the value of the slip length leads to a decrease in oscillation amplitude. However, at a relatively small slip length, decreasing the value of $C a$ to 0.0375 (we have kept $W e$ fixed here, motivated by Fig. 16) makes the oscillation strong again, as shown in Fig. 17(a). In Fig. 17(b), we show the angle that the interface makes with the tube wall as a function of the distance to the moving contact line, for the cases shown in Fig. 17(a), at an instance when the moving contact lines velocities are approximately equal. An apparent contact angle can be defined by extrapo-

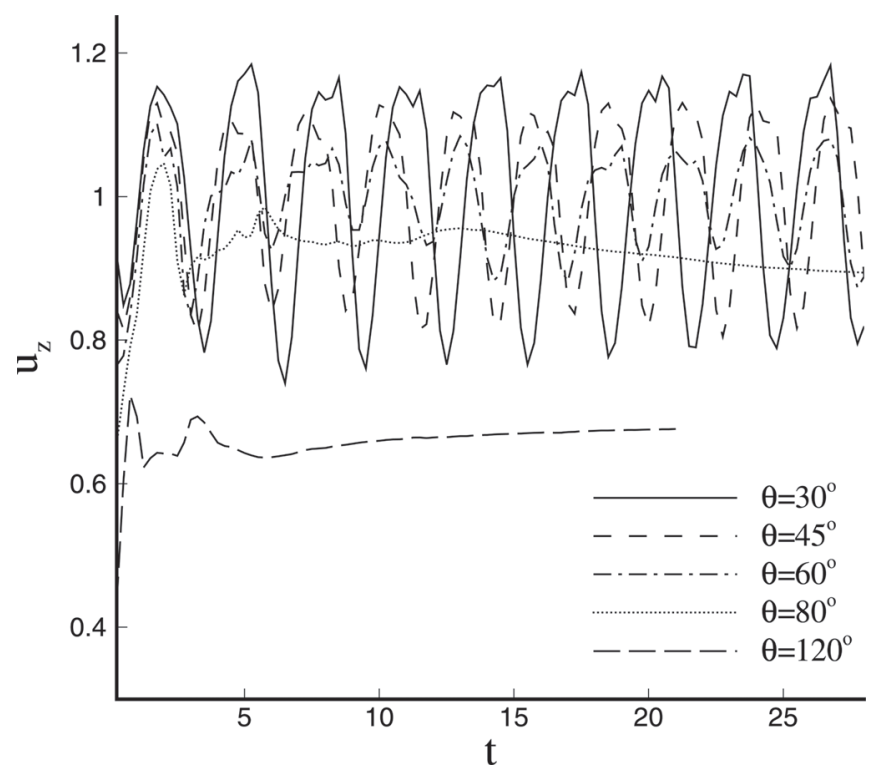

FIG. 14. Time evolution of the contact line velocity along the tube wall for different contact angles at $R e=450, C a=0.05$.

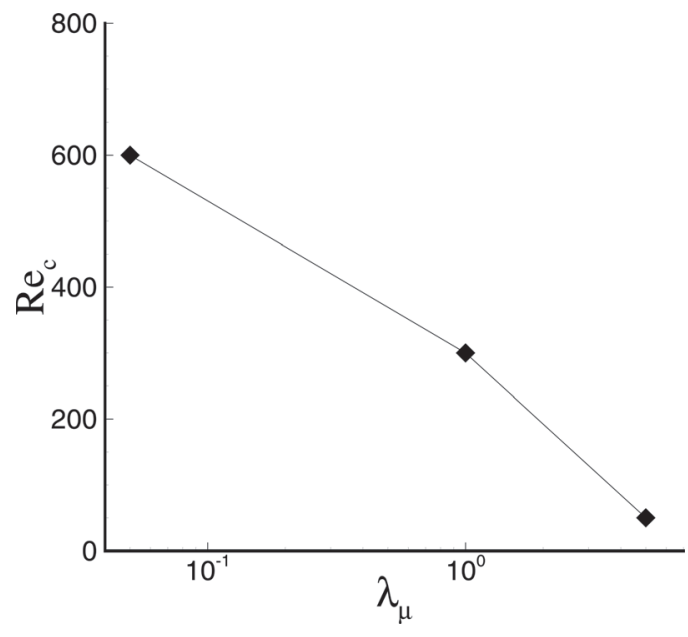

FIG. 15. Critical Reynolds number for jetting transition as a function of viscosity ratio for $C a=0.05, \vartheta=45^{\circ}$.

lating the linear region near the contact line to $s=0$. This apparent angle is found to increase upon reducing the slip length. From the results in Fig. 14, it may be expected that an increase in apparent angle would suppress the oscillation of the interface. When $C a$ is reduced to 0.0375 at $\varepsilon=0.004$, it is found in Fig. 17(b) that the apparent angle (thus defined) decreases to a value close to that for $C a=0.05$ and $\varepsilon=0.02$, and strong oscillations with a similar amplitude appears again. This is also supported by analytical theories of contact-line motion. For instance, the theory of $\mathrm{Cox}^{29}$ relates an apparent angle to the static angle through the relation,

$$
g\left(\theta_{a}\right)=g\left(\theta_{w}\right)+C a \cdot \ln \left(\frac{l_{m a}}{l_{m i}}\right)
$$

where $g$ is the integration function, $l_{m a}$ is the macroscopic length scale (e.g., tube radius), and $l_{m i}$ represents the microscopic length scale which is usually set as the slip length. Of course, this theory is for creeping flows, but in a later study, $\mathrm{Cox}^{51}$ argued that for rapid spreading, a viscous sublayer

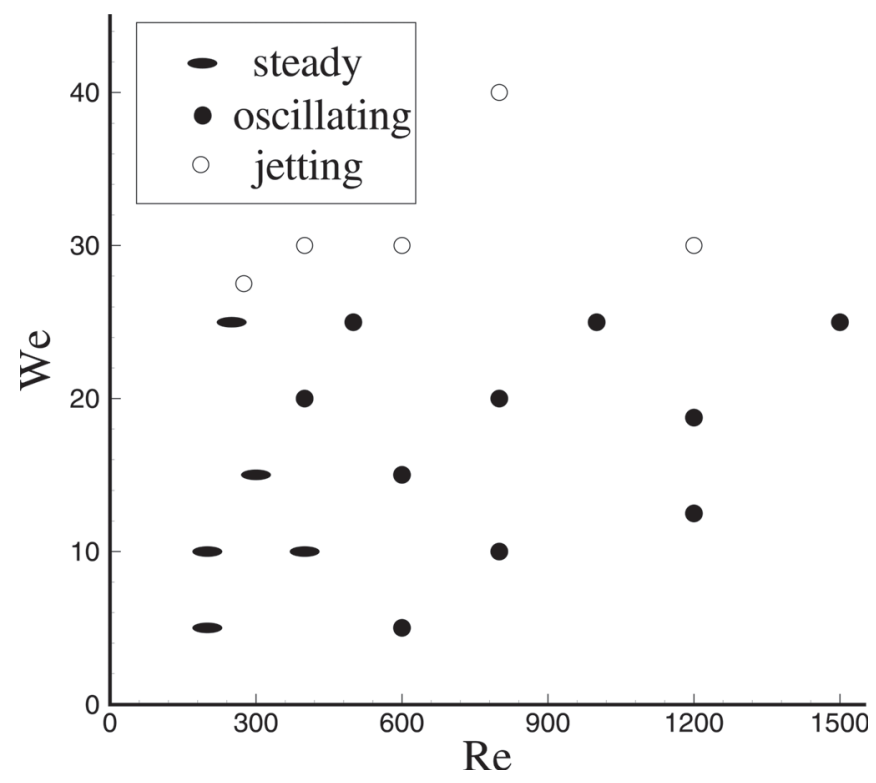

FIG. 16. Phase diagram of different flow states for $\vartheta=45^{\circ}$. 

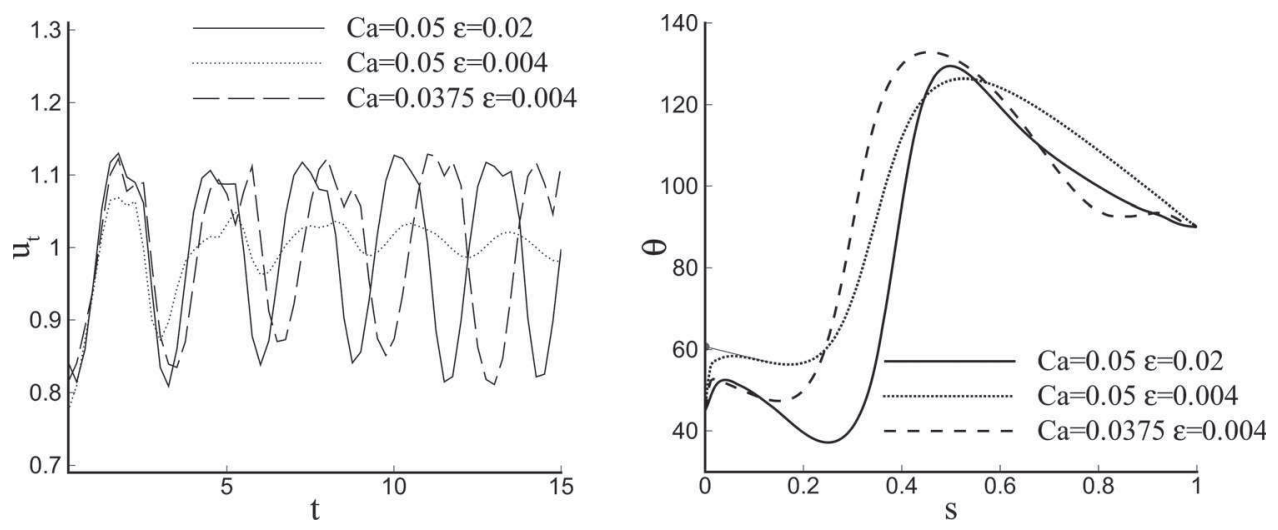

FIG. 17. (a) Time evolution of the contact line velocity along the tube wall for different slip lengths for $\mathrm{We}=22.5$ and (b) angle between interface and vertical tube wall as a function of interfacial distance to the moving contact line. (between an inertial sublayer and the wall) can be identified, and that the angle at the outer edge of that layer satisfies Eq. (2) above if the argument of the log term is divided by a Reynolds number. The present simulations do not quite meet the criteria for which this theory was derived $(R e \gg 1$ and $C a \ll 1$ whilst $\left(l_{m i} / l_{m a}\right) R e \ll 1$; this last condition is not met herein, which leads to a negative log term in the analogue of Eq. (2)), and we therefore use Eq. (2) and return to this issue below. Now, a reduction in slip length is seen to increase the second term on the right-hand-side of Eq. (2) and therefore increases the apparent angle, while decreasing $C a$ has the opposite effect. If we compare the value of $C a \cdot \ln \left(\frac{a}{\varepsilon}\right)$ for the two cases with strong oscillation in Fig. 17, it is found that these values are 0.20 and 0.21 for $C a=0.05$ and 0.0375 , respectively, which are very close. An additional factor of $R e$ would not significantly change the argument, since $R e$ only changes from 450 to 600 in the $\log$ term. This would suggest that for realistic conditions, e.g., $\epsilon=0.0001$, a capillary number which can keep the value of $C a \cdot \ln \left(\frac{a}{\varepsilon}\right)$ close to 0.2 (giving $C a=0.022$ ) would be expected to lead to an oscillating state of the interface for conditions in Fig. 17 ( $W e=22.5$ and $\vartheta=45^{\circ}$ ). As this would correspond to a change in $R e$ from 600 to about 1000, which would suggest that the argument in the log term in the equivalent of Eq. (2) for the viscous sublayer of $\mathrm{Cox}^{51}$ would not drastically alter due to a change in the value of $R e$, thereby supporting the assumptions made regarding the use of Eq. (2). It should be noted that the Navier-slip condition has been used here along the entire pipe wall, not only in the proximity of the moving contact line, as is the case of Cox. ${ }^{29,51} \mathrm{We}$ have carried out simulations wherein the Navier-slip condition is only applied in a small region within one slip length from the contact line, and the result (not shown) indicates that there is only a modest quantitative difference (which of course would be smaller if a slightly larger slip region is used). Similar insensitivity has been observed in the work of Bazhlekov and Chesters. ${ }^{33}$

In the jetting regime, we find that a smaller slip length results in a lower velocity of the moving contact line and so that more liquid will go to the tube centre to form a stronger jet. This leads to a lower critical Reynolds number for transition to jetting.

\section{E. Flow resistance}

The present displacement flow in a tube may provide useful information for the modelling of multiphase flows in porous media consisting of tubes networks. A relatively simple multiphase model ${ }^{52}$ is based on Darcy's law, in which fully developed tube flow is assumed and the flow resistance is proportional to the wetted length. More developed models ${ }^{7,12}$ take into account the effect of the interface, and the capillary pressure is always assumed as a constant.

In the present study, we have calculated the wall shear force from the simulation results and normalized it by the force obtained from Darcy's law, ${ }^{52}$ resulting in a relative flow resistance,

$$
\text { Relative flow resistance }=\frac{\int \sigma_{r z} d l}{\sigma_{r z}^{A} \cdot l},
$$

where $\sigma_{r z}$ and $\sigma_{r z}^{A}$ represent the wall shear stress from simulation and analytical solution and $l$ is the wetted length $l$. From the results presented in Fig. 18, it can be seen that the simplified model based on Darcy's law underestimates the flow resistance at the early stage of filling of the tube. This is because the wetted length is short and the shear force contribution from region near the contact line is relatively stronger. A strong oscillation in the flow resistance can be observed when the liquid interface is in the oscillating regime, which may have significant consequences for porous

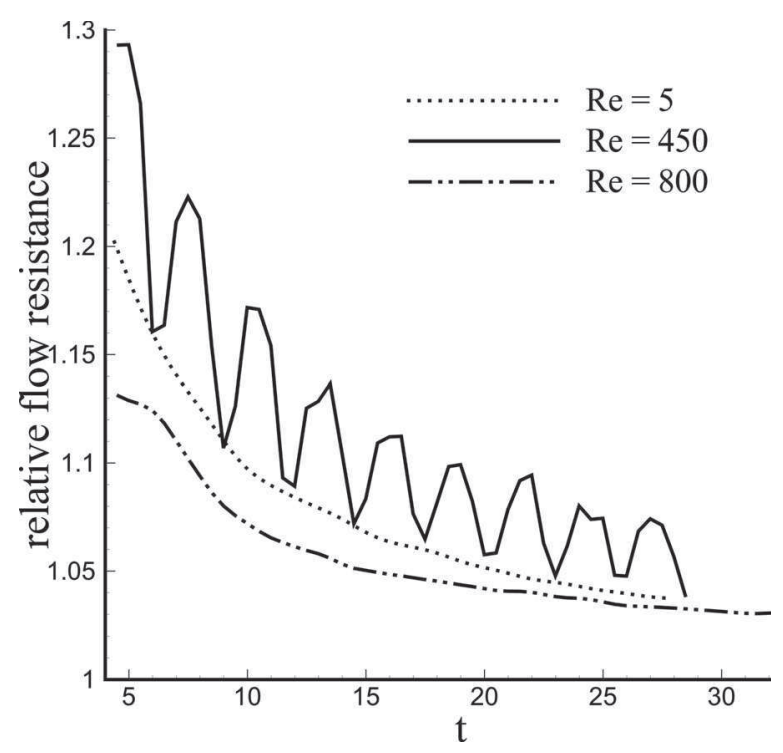

FIG. 18. Time evolution of relative flow resistance at different flow regimes for $C a=0.05, \vartheta=45^{\circ}$. 
media consisting of tubes networks when the flow rate in individual capillaries is rather large. The oscillation of flow resistance due to the inertia effects may have to be taken into account when developing advanced network models for high speed flow through porous media. ${ }^{18}$

\section{CONCLUSION AND DISCUSSION}

We have investigated inertial effects in the flow of a fluid displacing another fluid in a tube, using a slip-length-based level-set method. Three different regimes are found for cases where the contact angle is small when increasing a Reynolds number, $R e$ (whilst keeping other parameters constant). At relatively low $R e$, the meniscus shape reaches a steady state, and the interfacial curvature at the tube centre changes from concave into convex with increasing $R e$. At a critical value of $R e$, the results indicate that a supercritical Hopf bifurcation occurs, beyond which the flow becomes unsteady: the interface oscillates periodically and then quasi-periodically upon a further increase of the value of $R e$, and the interface evolves from simple-wavy to complex shapes with multiple wavy units. This state has not been observed previously, to our knowledge. Beyond a second critical value of $R e$, the displacing fluid forms a jet and the meniscus advances with a constant speed which decreases with $R e$. In the jetting regime, the interface shape remains steady at the tube centre and in the contact line region, whereas the region between these two regions is merely increasing its length continuously.

Further work in this area would certainly require an experimental confirmation of especially the oscillatory state reported here for the first time. Although the results presented here are for rather large values of a slip length, the results in Sec. III D suggest that a smaller value of $C a$ would be required for the oscillatory regime to be observed at realistically small slip length values. Also, it seems clear that a smaller slip length would lower the critical values of $R e$ for jetting to occur.

With regard to applications of the results presented herein, these seem at the moment most obvious in the area of modelling of locally high-speed invasion of porous media by a liquid displacing another fluid. Even such flows at otherwise low-Re could potentially still reach in some capillaries rather large $R e$ values if strong fingering phenomena occur, and the critical values of $R e$ for the various unsteady flow states reported in this study could serve as upper estimates, in the light of the slip length values used. Regarding the displacement efficiency, flows in the steady and oscillating regimes are similar since the interface moves on average with the mean inflow velocity, and the efficiency is higher than in the jetting regime where the contact line lags behind the interface front at the tube center. To increase the displacement efficiency, flow parameters (i.e., $R e, \theta, \lambda_{\mu}$ ) should be controlled to avoid the formation of jetting. Whether oscillations in the relation between pressure drop over a capillary and flow rate due to inertial effects might affect the formation of finger-type instabilities in networks would have to be the subject of further work in this area. Naturally, the present results would be representative of a class of porous media where the microstructure can reasonably be modelled by the geometry considered here; other media would require simulation/experiments on a two-phase front through a bed of spherical particles or cylindrical fibres, extending earlier single-phase flow work (e.g., Refs. 23-25).

\section{ACKNOWLEDGMENTS}

The authors acknowledge the financial support from EPSRC (Grant No. EP/E046029/1).

${ }^{1}$ D. A. de Sousa, E. J. Soares, R. S. de Queiroz, and R. L. Thompson, "Numerical investigation on gas displacement of a shear-thinning liquid and a visco-plastic material in capillary tubes," J. Non-Newtonian Fluid Mech. 144, 149 (2007).

${ }^{2}$ E. J. Soares and R. L. Thompson, "Flow regimes for the immiscible liquid-liquid displacement in capillary tubes with complete wetting of the displaced liquid,” J. Fluid Mech. 641, 63 (2009).

${ }^{3}$ Y. Dimakopoulos and J. Tsamopoulos, "Transient displacement of viscoplastic fluids by air in straight or suddenly constricted tubes," J. NonNewtonian Fluid Mech. 112, 43 (2003).

${ }^{4}$ Y. Dimakopoulos and J. Tsamopoulos, "Transient displacement of a Newtonian fluid by air in straight or suddenly constricted tubes," Phys. Fluids 15, 1973 (2003).

${ }^{5}$ Y. Dimakopoulos and J. Tsamopoulos, "On the gas-penetration in straight tubes completely filled with a viscoelastic fluid," J. Non-Newtonian Fluid Mech. 117, 117 (2004).

${ }^{6}$ Q. Kang, D. Zhang, and S. Chen, "Immiscible displacement in a channel: Simulations of fingering in two dimensions," Adv. Water Resour. 27, 13 (2004).

${ }^{7}$ J. Koplik and T. J. Lasseter, "Two-phase flow in random network models of porous media," SPEJ 25, 89 (1985).

${ }^{8}$ M. M. Dias and A. C. Payatakes, "Network models for two-phase flow in porous media. Part I. Immiscible microdisplacement of non-wetting fluids,” J. Fluid Mech. 164, 305 (1986).

${ }^{9}$ R. Lenormand, E. Touboul, and C. Zarcone, "Numerical models and experiments on immiscible displacements in porous media," J. Fluid Mech. 189, 165 (1988).

${ }^{10}$ M. Blunt and M. J. King, "Simulation and theory of two-phase flow in porous media," Phys. Rev. A 46, 7680 (1992).

${ }^{11}$ P. E. Øren, S. Bakke, and O. J. Arntzen, "Extending predictive capabilities to network models," SPEJ 3, 324 (1998).

${ }^{12}$ M. Blunt, M. D. Jackson, M. Piri, and P. H. Valvatne, "Detailed physics, predictive capabilities, and macroscopic consequences for pore-network models of multiphase flow," Adv. Water Resour. 25, 1069 (2002).

${ }^{13}$ V. Joekar-Niasar, S. M. Hassanizadeh, and H. K. Dahle, "Nonequilibrium effects in capillarity and interfacial area in two-phase flow: Dynamic porenetwork modelling," J. Fluid Mech. 655, 38 (2010).

${ }^{14} \mathrm{E}$. Ramé, "On an approximate model for the shape of a liquid-air interface receding in a capillary tube," J. Fluid Mech. 342, 87 (1997).

${ }^{15}$ D. Quéré, “Inertial capillarity,” Europhys. Lett. 39, 533 (1997).

${ }^{16}$ N. Fries and M. Dreyer, "The transition from inertial to viscous flow in capillary rise," J. Colloid Interface Sci. 327, 125 (2008).

${ }^{17}$ M. Stange, M. E. Dreyer, and H. J. Rath, "Capillary driven flow in circular cylindrical tubes," Phys. Fluids 15, 2587 (2003).

${ }^{18}$ R. A. Wooding and H. J. Morel-Seytoux, "Multiphase fluid flow through porous media,” Annu. Rev. Fluid Mech. 8, 233 (1976).

${ }^{19}$ F. A. L. Dullien, "Single phase flow through porous media and pore structure," Chem. Eng. J. 10, 1 (1975).

${ }^{20}$ I. F. Macdonald, M. S. El-Sayed, K. Mow, and F. A. L. Dullien, "Flow through porous media-the Ergun equation revisited," Ind. Eng. Chem. Fundam. 18, 199 (1979).

${ }^{21}$ A. Dybbs and R. V. Edwards, "A new look at porous media fluid mechanics-Darcy to turbulent," in Fundamentals of Transport Phenomena in Porous Media, edited by J. Bear and M. Y. Corapcioglu (Martinus Nijhoff Publishers, Dordrecht, 1984).

${ }^{22}$ E. Aker, K. J. Måløy, A. Hansen, and G. G. Batrouni, “A two-dimensional network simulator for two-phase flow in porous media," Transp. Porous Media 32, 163 (1998).

${ }^{23}$ R. J. Hill, D. L. Koch, and A. J. C. Ladd, "The first effects of fluid inertia on flows in ordered and random arrays of spheres," J. Fluid Mech. 448, 213 (2001). 
${ }^{24}$ R. J. Hill, D. L. Koch, and A. J. C. Ladd, "Moderate-Reynolds-number in ordered and random arrays of spheres," J. Fluid Mech. 448, 243 (2001).

${ }^{25}$ P. D. M. Spelt, T. Selerland, C. J. Lawrence, and P. D. Lee, "Flows of inelastic non-Newtonian fluids through arrays of aligned cylinders. Part 2. Inertial effects for square arrays," J. Eng. Math. 51, 81 (2005).

${ }^{26}$ R. L. Hoffman, "A study of the advancing interface," J. Colloid Interface Sci. 50, 228 (1975).

${ }^{27}$ R. T. Foister, "The kinetics of displacement wetting in liquid/liquid/solid systems,” J. Colloid Interface Sci. 136, 266 (1990).

${ }^{28} \mathrm{M}$. Fermigier and P. Jenffer, "An experimental investigation of the dynamic contact angle in liquid-liquid systems," J. Colloid Interface Sci. 146, 226 (1991).

${ }^{29}$ R. G. Cox, "The dynamics of the spreading of liquids on a solid surface. Part 1. Viscous flow," J. Fluid Mech. 168, 169 (1986).

${ }^{30}$ L. M. Hocking, "The spreading of a thin drop by gravity and capillarity," Q. J. Mech. Appl. Math. 36, 55 (1983).

${ }^{31}$ L. M. Hocking, "Rival contact-angle models and the spreading of drops," J. Fluid Mech. 239, 671 (1992).

${ }^{32}$ J. Lowndes, "The numerical simulation of the steady movement of a fluid meniscus in a capillary tube," J. Fluid Mech. 101, 631 (1980).

${ }^{33}$ I. B. Bazhlekov and A. K. Chesters, "Numerical investigation of the dynamic influence of the contact line region on the macroscopic meniscus shape," J. Fluid Mech. 329, 137 (1996).

${ }^{34}$ E. B. Dussan, V. E. Ramé, and S. Garoff, "On identifying the appropriate boundary conditions at a moving contact line: An experimental investigation,” J. Fluid Mech. 230, 97 (1991).

${ }^{35}$ W. Boender, A. K. Chesters, and A. J. J. van der Zanden, “An approximate analytical solution of the hydrodynamic problem associated with an advancing liquid-gas contact line, Int. J. Multiphase Flow 17, 661 (1991).

${ }^{36}$ P. D. M. Spelt, "A level-set approach for simulations of flows with multiple moving contact lines with hysteresis," J. Comput. Phys. 207, 389 (2005).

${ }^{37}$ P. D. M. Spelt, "Shear flow past two-dimensional droplets pinned or moving on an adhering channel wall at moderate Reynolds numbers: A numerical study," J. Fluid Mech. 561, 439 (2006).

${ }^{38}$ H. Ding, P. D. M. Spelt, and C. Shu, "Diffuse interface mode for incompressible two-phase flows with large density ratios," J. Comput. Phys. 226, 2078 (2007).
${ }^{39}$ H. Ding and P. D. M. Spelt, "Inertial effects in droplet spreading: A comparison between diffuse interface and level-set simulations," J. Fluid Mech. 576, 287 (2007).

${ }^{40}$ M. Sussman, A. S. Almgren, J. B. Bell, P. Colella, L. H. Howell, and M. L. Welcome, "An adaptive level set approach for incompressible twophase flows,” J. Comput. Phys. 148, 81 (1999).

${ }^{41}$ G. Russo and P. Smereka, "A remark on computing distance functions," J. Comput. Phys. 163, 51 (2000).

${ }^{42}$ X. D. Liu, S. Osher, and T. Chan, "Weighted essentially non-oscillatory schemes,” J. Comput. Phys. 115, 200 (1994).

${ }^{43}$ M. Renardy, Y. Renardy, and J. Li, "Numerical simulation of moving contact line problems using a volume-of-fluid method," J. Comput. Phys. 171, 243 (2001).

${ }^{44}$ S. Afkhami, S. Zaleski, and S. M. Bussmann, "A mesh-dependent model for applying dynamic contact angles to VOF simulations," J. Comput. Phys. 228, 5370 (2009).

${ }^{45} \mathrm{~K}$. Tsiveriotis and R. A. Brown, "Solution of free-boundary problems using finite-element/Newton methods and locally refined grids: Application to analysis of solidification microstructure," Int. J. Numer. Methods Fluids 16, 827 (1993).

${ }^{46}$ N. Chatzidai, A. Yanousakis, Y. Dimakopoulos, and J. Tsamopoulos, “On the elliptic mesh generation in domains containing multiple inclusions and undergoing large deformations," J. Comput. Phys. 228, 1980 (2009).

${ }^{47}$ A.-L. Biance, C. Clanet, and D. Queré, "First steps in the spreading of a liquid droplet," Phys. Rev. E 69, 016301 (2004).

${ }^{48}$ X. Bian, M. Perlin, W. W. Schultz, and M. Agarwal, "Axisymmetric slosh frequencies of a liquid mass in a circular cylinder," Phys. Fluids 15, 3659 (2003).

${ }^{49}$ J. A. Marsh, S. Garoff, and E. B. Dussan, "Dynamic contact angles and hydrodynamics near a moving contact line," Phys. Rev. Lett. 70, 2778 (1993).

${ }^{50}$ J. Eggers and H. A. Stone, "Characteristic lengths at moving contact lines for a perfectly wetting fluid: The influence of speed on the dynamic contact angle," J. Fluid Mech. 505, 309 (2004).

${ }^{51}$ R. G. Cox, "Inertial and viscous effects on dynamic contact angles," J. Fluid Mech. 357, 249 (1998).

${ }^{52}$ I. Fatt, "The network model of porous media III. Dynamic properties of networks with tube radius distribution," Trans. AIME 181, 164 (1956). 